\title{
Editorial
}

\section{Submitting an article on gastric cancer: to which journal?}

\author{
YASUHIRO KODERA \\ Department of Surgery II, Nagoya University Graduate School of Medicine, 65 Tsurumai-cho, Showa-ku, Nagoya 466-8550, Japan
}

This is the 6th year since the publication of the first issue of the journal Gastric Cancer - a unique journal devoted wholly to articles on gastric neoplasms. It is a great pleasure for the editorial board members that the number of manuscripts submitted to the journal has increased over the past few years. There is almost always a struggle for a new journal to gather a sufficient number of high-quality papers for publication, and Gastric Cancer has been no exception. For this reason particularly, an article by Lunet et al. [1] that assessed the trend in publications on gastric cancer caught our eye.

Of 14437 cancer articles that Lunet and his colleagues found in PubMed, 3.1\% addressed the subject of stomach cancer. This proportion has not changed over the past 20 years and is far below its expected share, given the high incidence of this type of cancer in the world. There has been a remarkable increase in the proportion of articles from Asia over the past 2 decades, compensating for the marked decrease in articles from the United States, where the incidence of gastric cancer has declined substantially. In the United States, the prognosis of gastric cancer has remained dismal despite substantial expenditure of funding for research on multimodal treatments, and this may also be the reason that the motivation to combat this disease has waned. Professor L.P. Leichman confessed at the annual meeting of the American Society of Clinical Oncology in 2000 that no further trials on neoadjuvant chemotherapy, the most promising strategy at that time for resectable advanced gastric cancer, had been planned in the United States because it was unlikely to be worth the expense. The incidence of gastric cancer is high in developing countries, where the economic resources for cancer investigation are limited in comparison with those in North America or Western Europe, and this may well be reflected in the number of articles published.

Offprint requests to: Y. Kodera
Interestingly, the proportion of articles addressing the etiology and biology of gastric cancer has increased significantly (from $29 \%$ to $56 \%$ ) over the past 2 decades. There is a situation in Japan, a major contributor to the literature on gastric cancer in the period 20002002 , that may be one of the reasons for this phenomenon. Gastric cancer being a common disease in Japan, it is not treated exclusively at specialized centers. Consequently, active and capable surgeons at university hospitals rarely perform more than 50 gastrectomies per year. However, it has become increasingly difficult for data from retrospective case series to find their way to the international journals such as those evaluated by Lunet et al. These journals look for new evidence that challenges the standard of care, evidence that can be obtained only through a prospective clinical trial with adequate study design. A well-designed clinical trial calls for a large sample, which can be accrued only through a multi-institutional study. It is unlikely that surgeons in low-volume university hospitals can play a leading role in large trials. However, a bank of snapfrozen tissues can be established with a few years of effort, and this could be a start for arrays of studies using methods in molecular biology. Sadly, therefore, original contributions from most surgeons and gastroenterologists at the university hospitals come from the bench and not from the bedside. Moreover, the majority of surgeons outside of university hospitals are not motivated to write articles in a foreign language.

Another important factor that may influence the acceptance of articles submitted is the editorial policy of scientific journals. This may be a crucial issue for those who specialize in gastric carcinoma, given that most highly regarded journals are published in the United States or Western Europe where, again, the incidence of gastric cancer has declined. It is unlikely that the editors and subscribers expect these journals to be filled with articles on gastric carcinoma, which, for many of them, may be a disease of the past. Because curative treatment 
of gastric cancer remains primarily surgical, it could have been more interesting and informative if the authors had looked more specifically into leading journals on surgery, along with those on general and experimental oncology that they had explored in detail. Scrutinizing surgery journals might have revealed the fact that an important issue in one community may not be fully understood or appreciated in another. For instance, no less than $50 \%$ of gastric cancer patients in high-volume Japanese hospitals have T1-stage disease, for which numerous minimally invasive approaches have been reported and discussed to ensure better quality of life for the patients without compromising curability [2]. However, these procedures are more or less irrelevant in Western countries where early-stage gastric cancer is a rarity.
All in all, the article by Lunet et al. illustrates the need for an international journal devoted to gastric cancer and peer-reviewed by true experts in the field. Under such circumstances, Gastric Cancer will play an important role, and I am convinced that a greater number of investigators will have an interest in the journal.

\section{References}

1. Lunet N, Carvalho R, Barros H. An appraisal of gastric cancer research in cancer journals. Gastric Cancer 2004;7:172-5.

2. Yokota T, Ishiyama S, Saito T, Teshima S, Shimotsuma M, Yamauchi $\mathrm{H}$. Treatment strategy of limited surgery in the treatment guidelines for gastric cancer in Japan. Lancet Oncol 2003;4: 423-8. 